\section{TRPping up overactive bladder}

\section{By Chris Cain, Staff Writer}

A collaboration between researchers at Hydra Biosciences Inc. and Catholic University Leuven has found that antagonizing the ion channel TRPV4 could provide a new mechanism to treat overactive bladder. ${ }^{1}$ The biotech expects that blocking the target will elicit fewer side effects than marketed anticholinergic therapies.

Overactive bladder develops when the nervous system is unable to properly control bladder muscle contraction. Its causes range from systemic neurological disorders like multiple sclerosis (MS) to localized inflammation of the bladder in the case of urinary tract infections. The majority of approved drugs for overactive bladder antagonize muscarinic acetylcholine receptors, which regulate the contraction of smooth muscle in the bladder.

However, because these receptors are found throughout the body, the antagonists can cause a host of side effects, including blurred vision, dry mouth and constipation.

The first clue that TRPV4 (transient receptor potential vanilloid 4; VRL2) could be a therapeutic target came in 2007, when researchers including Thomas Voets, professor of molecular cell biology at the Catholic University Leuven, showed lower bladder function in Trpv4 mutant mice than in wild-type controls. ${ }^{2}$

Voets began the current study by showing that in a model of urinary bladder inflammation, Trpv 4 mutant mice had less intense symptoms of overactive bladder than wild-type controls. But to nail down the therapeutic potential of antagonizing TRPV4, he needed a small molecule inhibitor to probe the channel's role in urinary incontinence.

This led to the collaboration with Hydra, which develops small molecules that target ion channels. The company had recently synthesized HC067047, a potent and selective TRPV4 antagonist, which Hydra thought could be useful for a variety of indications including pain, osteoporosis and overactive bladder.

Voets and Hydra now have shown that in mouse and rat models of urinary bladder inflammation, HC-067047 decreased urination frequency and increased urine volume compared with vehicle controls.

There were no systemic adverse effects on body temperature, heart rate, fluid intake, thermal selection behavior or motor coordination.

Results were published in the Proceedings of the National Academy of Sciences.

"Antimuscarinics target bladder contraction, as do most drugs in development for urinary disorders," noted Christopher Fanger, senior director of lead discovery at Hydra. "Blocking TRPV4, on the other hand, would target the problem at its origin: sensing the filling state of the bladder. By focusing on the unpleasant sensation itself rather than bladder voiding, we think TRPV4 blockers may be preferable to the patient."

"What this Belgian team has demonstrated is the possibility that TRPV4 inhibition can be accomplished therapeutically without lethal side effects-a relatively surprising finding," said Wolfgang Liedtke, assistant professor and attending physician at Duke University. He was the first author on the initial description of TRPV4 in Cell ten years ago. ${ }^{3}$

Side effects were a primary concern, noted Liedtke, because previous studies published by GlaxoSmithKline plc showed that activating Trpv4 led to severe cardiovascular side effects and death in rats, raising questions about the safety of modulating TRPV $4 .^{4}$

The current work represents the first time that researchers have tried to block the target in vivo.

In addition, Liedtke said, there are early indications that inhibiting TRPV4 could have other clinical uses, including the treatment of bronchitis or colitis. He cited studies showing that TRPV4 activation can lead to colitis and that TRPV4 regulates ciliary clearance function in mammalian airway epithelia.,

When asked about the potential for side effects, Hydra suggested that at the dose used there may be complete inhibition in the bladder but not necessarily in other tissues.

GSK filed a patent application covering its own TRPV4 antagonists. The pharma declined to comment on the status of the application or whether it has any TRPV4 modulators in development.

Hydra did say it needs to modify HC-067047 to generate a candidate that is suitable for clinical development. HC-067047 is not sufficiently orally bioavailable, and the company believes an injectable medication would be undesirable for an indication such as overactive bladder.

Hydra has filed for patents covering HC-067047 and hopes to partner its TRPV4 program, which includes HC-067047 and other TRPV4modulating compounds.

Cain, C. SciBX 3(44); doi:10.1038/scibx.2010.1311

Published online Nov. 11, 2010

\section{REFERENCES}

1. Everaerts, W. et al. Proc. Natl. Acad. Sci. USA; published online Oct. 18, 2010; doi:10.1073/pnas.1005333107

Contact: Christopher M. Fanger, Hydra Biosciences Inc., Cambridge, Mass.

e-mail: cfanger@hydrabiosciences.com

Contact: Thomas Voets, Catholic University Leuven, Leuven, Belgium e-mail: thomas.voets@med.kleuven.be

2. Gevaert, T. et al. J. Clin. Invest. 17, 3453-3462 (2007)

3. Liedtke, W. et al. Cell 103, 525-535 (2000)

4. Willette, R.N. et al. J. Pharmacol. Exp. Ther. 326, 443-452 (2008)

5. D'Aldebert, E. et al. Gastroenterology; published online Sept. 30, 2010; doi:10.1053/j.gastro.2010.09.045

6. Lorenzo, I.M. et al. Proc. Natl. Acad. Sci. USA 105, 12611-12616 (2008)

COMPANIES AND INSTITUTIONS MENTIONED

Catholic University Leuven, Leuven, Belgium

Duke University, Durham, N.C.

GlaxoSmithKline plc (LSE:GSK; NYSE:GSK), London, U.K.

Hydra Biosciences Inc., Cambridge, Mass. 